\title{
In vivo pre-activation of monocytes in patients with axial spondyloarthritis
}

Kristina Conrad ${ }^{1}$, Peihua $\mathrm{Wu}^{2}$, Joachim Sieper ${ }^{1,2}$ and Uta Syrbe ${ }^{1,2^{*}}$

\begin{abstract}
Introduction: Innate immune responses, including monocyte functions, seem to play an important role in the pathogenesis of axial spondyloarthritis (axSpA). Therefore, we characterized the phenotype and functional state of monocytes of patients with axSpA.

Methods: Fifty-seven patients with axSpA, 11 patients with rheumatoid arthritis (RA), and 29 healthy controls were included in the study. We determined the percentage of classic, intermediate, and non-classic monocytes according to CD14 and CD16 expression and the expression of Toll-like receptor (TLR) 1, 2, and 4 in whole blood by flow cytometry. The percentage of monocytes producing interleukin (IL)-1 beta, IL-6, tumor necrosis factor alpha (TNFa), IL-12/23p40, and IL-1 receptor antagonist (IL-1 ra) was detected by flow cytometry after stimulation of whole blood without and with different TLR and nucleotide-binding oligomerization domain ligands-i.e., lipopolysaccharide (LPS), fibroblast-stimulating lipopeptid-1, $\mathrm{PAM}_{3} \mathrm{CSK}_{4}$, and muramyl dipeptide (MDP)—for $5 \mathrm{~h}$. IL-10 production was measured after $18 \mathrm{~h}$ of stimulation in supernatants by enzyme-linked immunosorbent assay.

Results: In patients with axSpA but not patients with RA, we found higher frequencies of classic monocytes than in controls (median of $90.4 \%$ versus $80.4 \%, P<0.05$ ), higher frequencies of monocytes spontaneously producing IL-1 beta and IL-1ra $(P<0.05)$, and a higher percentage of monocytes producing IL-1 beta after MDP stimulation $(P<0.05)$. Elevated cytokine production was confined to axSpA patients under conventional therapy (non-steroidal anti-inflammatory drugs) and not found in patients under TNFa inhibitor treatment. The LPS-induced production of IL-6 and IL-10 was lower in axSpA patients compared with controls $(P<0.05)$. Monocytic TLR expression was unaffected in patients with axSpA.
\end{abstract}

Conclusion: Enhanced spontaneous and MDP-induced cytokine secretion by monocytes suggests in vivo pre-activation of monocytes in axSpA patients under conventional therapy which is reverted under TNF inhibitor treatment.

\section{Introduction}

Axial spondyloarthritis (AxSpA) is the prototypic form of spondyloarthritis, a group of related diseases comprising AxSpA, reactive arthritis, arthritis related to inflammatory bowel disease, psoriasis, and uveitis [1]. AxSpA, nowadays subdivided into non-radiographic axSpA and ankylosing spondylitis (AS), is characterized by inflammation within the axial skeleton, predominately of the sacroiliac joints [1].

\footnotetext{
* Correspondence: uta.syrbe@charite.de

${ }^{1}$ Charité-Universitätsmedizin Berlin, CBF, Medizinische Klinik für

Gastroenterologie, Infektiologie und Rheumatologie, Hindenburgdamm 30, Berlin 12203, Germany

Deutsches Rheumaforschungszentrum, Charitéplatz 1, Berlin 10117, Germany
}

(c) 2015 Conrad et al. This is an Open Access article distributed under the terms of the Creative Commons Attribution License (http://creativecommons.org/licenses/by/4.0), which permits unrestricted use, distribution, and reproduction in any medium, provided the original work is properly credited. The Creative Commons Public Domain Dedication waiver (http:// creativecommons.org/publicdomain/zero/1.0/) applies to the data made available in this article, unless otherwise stated. are still unclear. AxSpA is strongly associated with the major histocompatibility complex (MHC) class I molecule HLA-B27; however, the involvement of CD8 T-cell responses could not be proven so far. In fact, in the HLAB27 transgenic rat model, CD8 T cells are dispensable for disease induction [2]. Apart from presenting specific antigens to $\mathrm{CD} 8^{+} \mathrm{T}$ cells, HLA-B27 has the capability to induce endoplasmic reticulum stress [3]. In innate immune cells, this can trigger cytokine responses or modify the responsiveness of these cells to other stimulants, suggesting that innate immune functions might play a role in the pathogenesis of axSpA.

Monocytes belong to the innate, first-line defence against infections. They recognize bacterial structures by pattern 
recognition receptors, including Toll-like receptor (TLR) and nucleotide-binding oligomerization domain (NOD)like-receptors. Ligation of TLR or NOD receptors rapidly triggers production of proinflammatory cytokines such as interleukin-1 (IL-1), IL-6, and tumor necrosis factor alpha $(\mathrm{TNF} \alpha)$ in monocytes [4, 5]. Proinflammatory responses are tightly controlled by concomitant induction of antiinflammatory mediators, such as IL-1ra, which competes with IL-1 for binding to the IL-1 receptor 1 [6], and IL-10, which is produced by monocytes at later time points after stimulation and downregulates TNF $\alpha$ production [7].

There are some reports that showed enhanced transcription and protein expression of inflammation-associated genes in blood monocytes of patients with AS, suggesting aberrant activation or disturbed responsiveness of monocytes in patients with axSpA $[8,9]$.

To further decipher potential involvement of monocytes in the pathogenesis of axSpA, we studied here the phenotype and function of monocytes from patients with axSpA. We performed a phenotypic analysis of monocytes and determined their spontaneous and TLR and NOD ligand-induced cytokine production.

\section{Methods}

\section{Study subjects}

Peripheral venous whole blood was collected in heparinised Vacutainer tubes (BD, Heidelberg, Germany) from 29 healthy donors (15/29 male, mean age of $35.3 \pm$ 8.3 years) and from 57 patients with axSpA (39/57 male, mean age of $38.0 \pm 10.6$ years) meeting the Assessment of Spondyloarthritis International Society criteria for axSpA, of whom 39 patients (68.4 \%) fulfilled the modified New York criteria for diagnosis of AS. Eleven patients $(2 / 11$ male, mean age of $59.7 \pm 18.8$ years) with rheumatoid arthritis (RA) were included as inflammatory controls. The number of patients and controls used for individual parts of the study (phenotypic analysis and cytokine analysis) is stated in each figure legend. Fiftythree $(92.9 \%)$ of the 57 patients with axSpA were HLAB27-positive. Thirty-four patients with axSpA received conventional treatment with continuous or on-demand non-steroidal anti-inflammatory drugs (NSAIDs), and 23 patients received TNF $\alpha$ inhibitors. None of the patients with axSpA received disease-modifying anti-rheumatic drugs (DMARDs). Ten out of eleven patients with RA received prednisolone, eight out of 11 received synthetic DMARD therapy (four methotrexate, two hydroxychloroquine, one sulfasalacine, and one methotrexate plus sulfasalacine), and two patients received treatment with biologics (one TNFo inhibitor and one anti-IL-6 receptor antibody). Disease activity was determined according to the Bath ankylosing spondylitis disease activity index (BASDAI) [10]. C-reactive protein (CRP) was determined at the routine clinical laboratory of the Charité.
The study was approved by the local ethical commission of the Charité. All patients and control subjects gave consent to the study.

Phenotypic characterization of peripheral blood monocytes To analyse the expression of phenotypic surface markers, $2 \mathrm{ml}$ of heparinized whole blood was incubated with $8 \mathrm{ml}$ of erythrocyte lysing buffer (Qiagen, Hilden, Germany) on ice to lyse erythrocytes. The remaining cells were stained with the respective antibodies against CD14 (clone M5E2 PerCPcy5.5; BD), CD16 (clone eBioCB16 APC; ebioscience, Frankfurt, Germany), HLA-DR (clone L243 fluorescein isothiocyanate (FITC); BD Heidelberg, Germany), CD64 (clone 10.1 PE; Dako, Glostrup, DK), CD80 (clone 2D10 PE; biolegend, San Diego, CA, USA), CD115 (clone 123A3-1B10 PE; ebioscience), and CD163 (clone GHI/61PE; BD). For analysis of TLR expression, $50 \mu \mathrm{l}$ of heparinized whole blood was incubated at room temperature with the respective antibodies against TLR1, i.e., CD281 (clone GD2.F4 TLR-1), TLR2, i.e., CD282 (clone TL2.1 TLR-2), and TLR4, i.e., CD284 (clone HTA125 TLR-4; all PE; ebioscience) in the dark for $15 \mathrm{~min}$. Afterwards, erythrocytes were lysed with fluorescence-activated cell sorting (FACS) lysing solution (BD) and leukocytes were analysed by flow cytometry by using a FACS Calibur from Becton Dickinson (San Jose, CA, USA) and FlowJo 7.6.4 Software.

In vitro stimulation of whole blood with Toll-like receptor and nucleotide-binding oligomerization domain ligands and intracellular cytokine detection

Heparinized peripheral blood $(1 \mathrm{ml})$ was stimulated for $5 \mathrm{~h}$ without or with the TLR $1 / 2$ ligand $\mathrm{PAM}_{3} \mathrm{CSK}_{4}$ (5 $\mu \mathrm{g} / \mathrm{ml}$ ), the TLR $2 / 6$ ligand fibroblast-stimulating lipopeptid-1 (FSL-1; $1 \mu \mathrm{g} / \mathrm{ml}$ ), the TLR 4 ligand lipopolysaccharide (LPS) (Escherichia coli O111:B4; $100 \mathrm{ng} / \mathrm{ml}$ ), and the NOD2 ligand muramyl dipeptide (MDP) $(2.5 \mu \mathrm{g} /$ $\mathrm{ml}$ ). All stimulants were purchased from invivoGen (San Diego, CA, USA).

To prevent cytokine secretion, $10 \mu \mathrm{g} / \mathrm{ml}$ brefeldin A (BFA) (Sigma-Aldrich, Steinheim, Germany) was added for the last $3 \mathrm{~h}$ of stimulation. After stimulation, erythrocytes were lysed with FACS lysing solution and the remaining cells were fixed by $2 \%$ paraformaldehyde (Roth, Karlsruhe, Germany). Intracellular cytokines and CD68 were stained after permeabilization in $0.5 \%$ saponin/phosphate-buffered saline buffer (Sigma-Aldrich, Steinheim, Germany) by using the following antibodies: anti-CD68-PerCPcy5.5 (clone Y1/82A; biolegend), antiIL-12/23p40 (clone eBioHP40; e-bioscience, Frankfurt, Germany), anti-TNF $\alpha$-FITC (clone Mab11; BD), anti-IL1 $\beta$-Alexa Fluor 647 (clone JK1B-1; biolegend), anti-IL-6FITC (clone MQ2-13A5; BD), and anti-IL-1ra-PE (clone AS17; BD). Cytokine expression was detected on a cellular level by using flow cytometric analysis. CD68 was 
used for identification of monocytes after stimulation, which yielded better separation of monocytes than CD14 after stimulation.

In vitro stimulation of whole blood with Toll-like receptor and nucleotide-binding oligomerization domain ligands and detection of interleukin-10 production by enzyme-linked immunosorbent assay

To determine IL-10 production, heparinised whole blood was mixed 1:2 with RPMI supplemented with $10 \%$ fetal calf serum and stimulated without and with the respective TLR and NOD ligands for $18 \mathrm{~h}$. Supernatants were harvested by centrifugation, snap-frozen, and stored at $-20{ }^{\circ} \mathrm{C}$ until measurement of IL-10 by enzyme-linked immunosorbent assay (ELISA) (BD OptEIA, Heidelberg, Germany).

\section{Statistical analysis}

Statistical analysis was performed by using GraphPad Prism 5 software (GraphPad Inc., La Jolla, CA, USA) and SPSS 19 software (IBM, Ehningen, Germany). Data are shown as individual measurements and medians. Non-parametric tests (Mann-Whitney $U$ test and Kruskal-Wallis test and Dunn's post test) were used for comparisons between groups. For variance analysis, we log-transformed data with uneven distribution and used linear regression analysis for metric variables and analysis of variance (ANOVA) for categorical variables.

\section{Results}

Expansion of classic monocytes in the peripheral blood of patients with axial spondyloarthritis

To analyse the distribution of monocyte subsets (i.e., classic, intermediate, and non-classic monocytes) within the peripheral blood of axSpA patients in comparison with healthy controls and RA patients as an inflammatory control, monocytes were gated according to forward and side scatter and expression of HLA-DR (Fig. 1a). In patients with axSpA, the percentage of classic monocytes $\left(\mathrm{CD} 14^{++} \mathrm{CD} 16^{-}\right)$was significantly higher than in healthy controls (median of $90.5 \%$ versus $80.4 \%, P<$ 0.05; Fig. 1b) whereas intermediate $\left(\mathrm{CD} 14^{++} \mathrm{CD} 16^{+}\right)$and non-classic $\left(\mathrm{CD} 14^{+} \mathrm{CD} 16^{+}\right)$subsets were reduced in patients with axSpA (Fig. 1b). In patients with RA, nonclassic monocytes were also reduced whereas differences in classic and intermediate monocyte subsets were not significantly different from controls. Subanalysis of axSpA patients grouped according to treatment into patients with conventional treatment (i.e., on demand or continuous NSAID treatment) or treatment with biologics showed significantly lower frequencies of intermediate $\mathrm{CD} 14^{++} \mathrm{CD} 16^{+}$ monocytes in patients under conventional as well as in patients treated with biologics compared with healthy controls, whereas the difference in frequencies of $\mathrm{CD} 14^{++}$ $\mathrm{CD}^{-}$monocytes reached statistical significance only in patients under standard therapy compared with healthy patients $(P<0.01)$ but not in patients under biological treatment (Fig. 1c).

The expression of HLA-DR as an antigen-presenting molecule, CD80 as a co-stimulatory molecule, CD163 as a scavenger receptor, CD64 as the Fc receptor, or CD115 as an activation marker on monocytes showed no difference between axSpA patients and controls $(P>0.05$; data not shown).

\section{In vivo pre-activation of monocytes of axial spondyloarthritis patients according to proinflammatory cytokine production}

To determine spontaneous as well as TLR and NOD ligand-induced production of cytokines by monocytes, we incubated whole blood of 42 patients with axSpA (32 male, 10 female; mean age of $37.2 \pm 9.8$ years), 8 patients with RA ( 2 male, 6 female; mean age of $59.7 \pm 18.8$ years; all patients on prednisolone and DMARD treatment, no treatment with biologics), and 26 controls (13 male; 13 female; mean age of $35.4 \pm 7.1$ years) without any stimulator or added $2.5 \mu \mathrm{g} / \mathrm{ml}$ MDP (NOD-2 ligand), $100 \mathrm{ng} /$ $\mathrm{ml}$ LPS (TLR4 ligand), $1 \mu \mathrm{g} / \mathrm{ml}$ FSL-1 (TLR2/6 ligand), or $5 \mu \mathrm{g} / \mathrm{ml} \mathrm{PAM}_{3} \mathrm{CSK}_{4}$ (TLR1/2 ligand). Cytokine expression was determined in monocytes which were identified according to CD68 expression (Fig. 2a).

The percentage of monocytes spontaneously producing IL-1 $\beta$ was significantly higher in axSpA patients compared with healthy controls (Fig. 2b). A similar nonsignificant trend toward higher production was observed for IL-6 and TNF $\alpha$ production in patients with axSpA, whereas the IL-12/23p40 production was not increased.

Stimulation with MDP and also stimulation with FSL1 elicited a higher cytokine production by monocytes, particularly of IL-1 $\beta$, but also to lesser extent of IL-6 and TNFa, in patients with axSpA (Fig. 2b). For instance, after MDP stimulation, $19.2 \%$ (median) of the monocytes of patients with axSpA versus $8 \%$ of monocytes of healthy controls $(P<0.01)$ produced IL-1 $\beta$. In contrast, IL-12/23 p40 production by monocytes was low (i.e., below $5 \%$ ) after MDP and FSL stimulation and was without difference between axSpA patients and healthy controls. In contrast, patients with RA did not show an elevated spontaneous or MDP- or FSL-induced production of proinflammatory cytokines, despite a minimal but significant increase in the percentage of IL-12/ 23p40-producting monocytes in unstimulated cultures. In contrast, no difference was observed between axSpA patients and controls in IL- $1 \beta$ and TNF $\alpha$ production in response to LPS, which elicited the highest cytokine response in monocytes. In patients with axSpA, more than $80 \%$ of blood monocytes produced IL- $1 \beta$ in response to LPS and about $60 \%$ produced TNF $\alpha$, which was not different from controls. Also, on a per-cell basis, LPS 

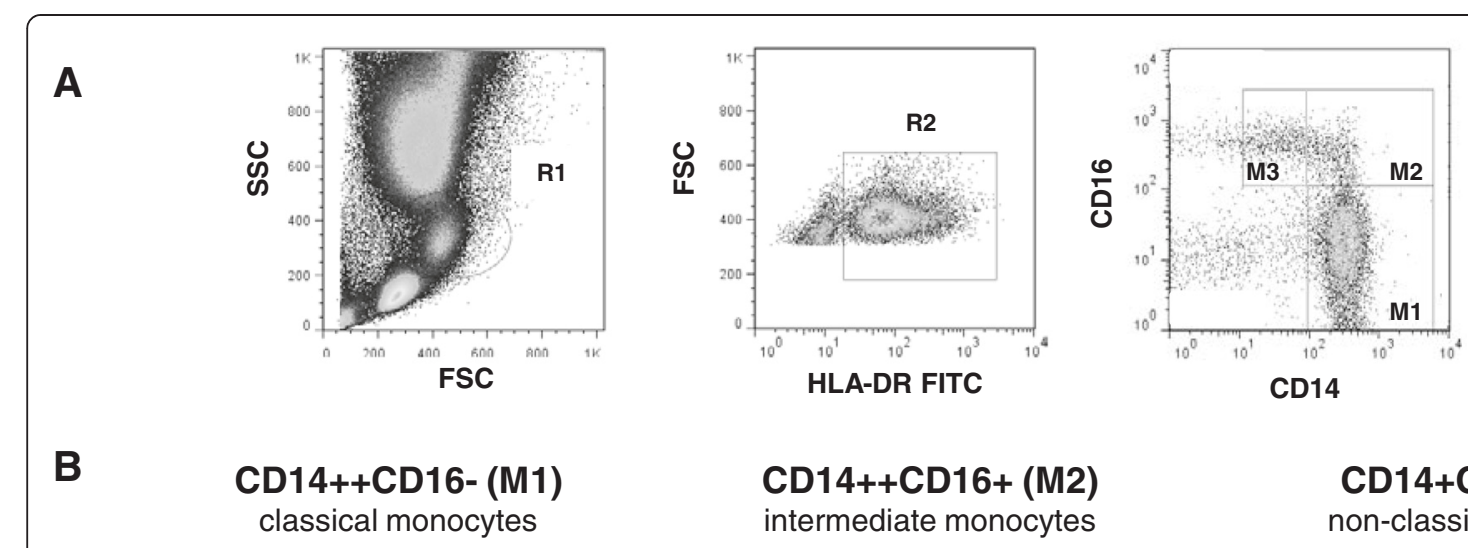

CD14++CD16+ (M2)

intermediate monocytes
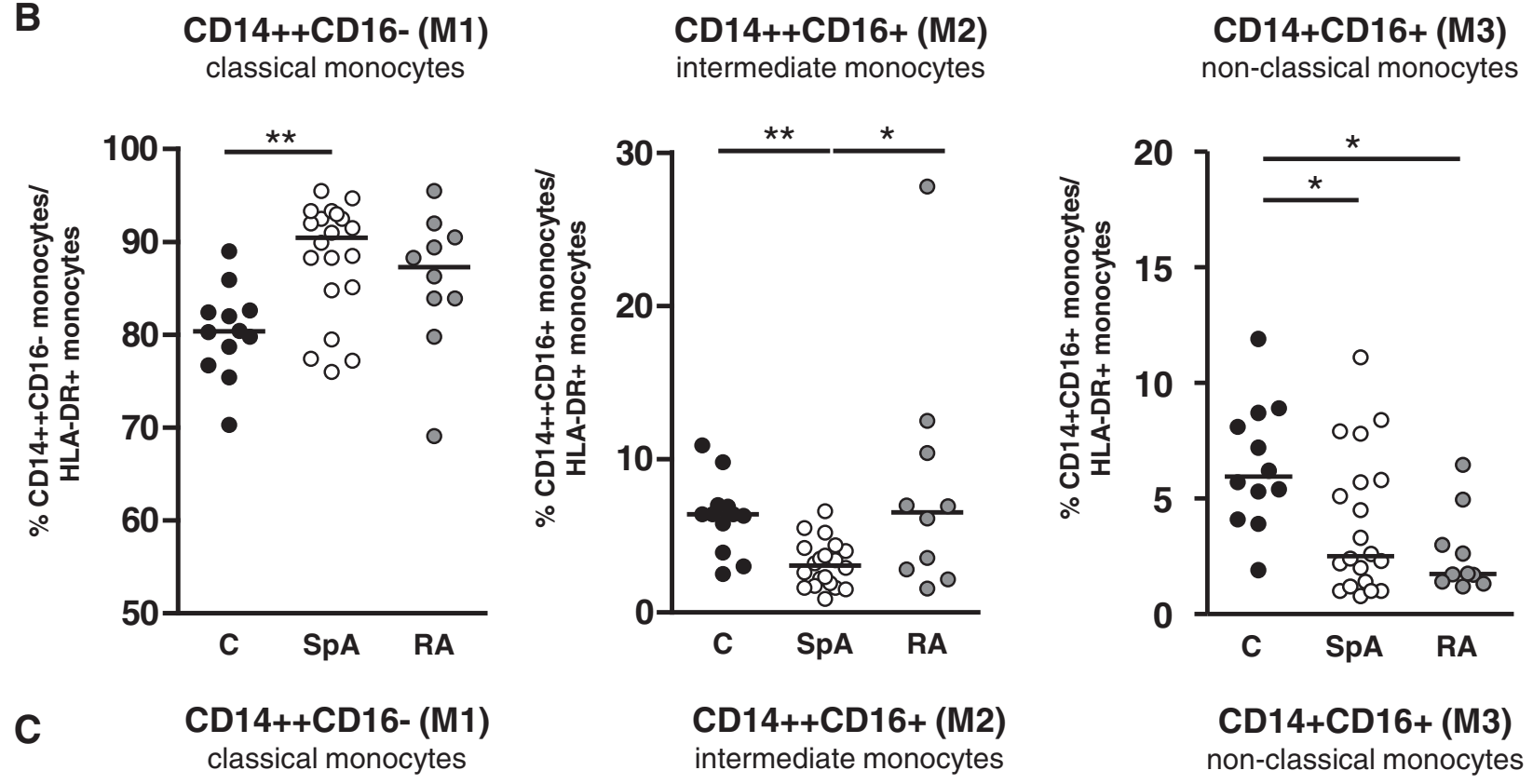
intermediate monocytes
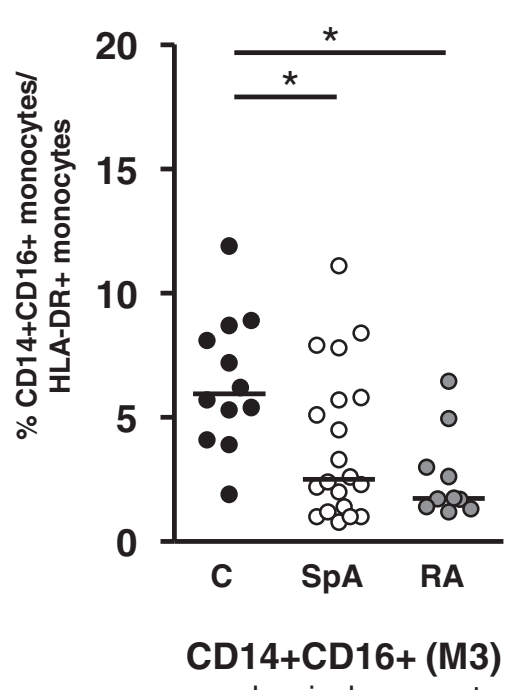

non-classical monocytes
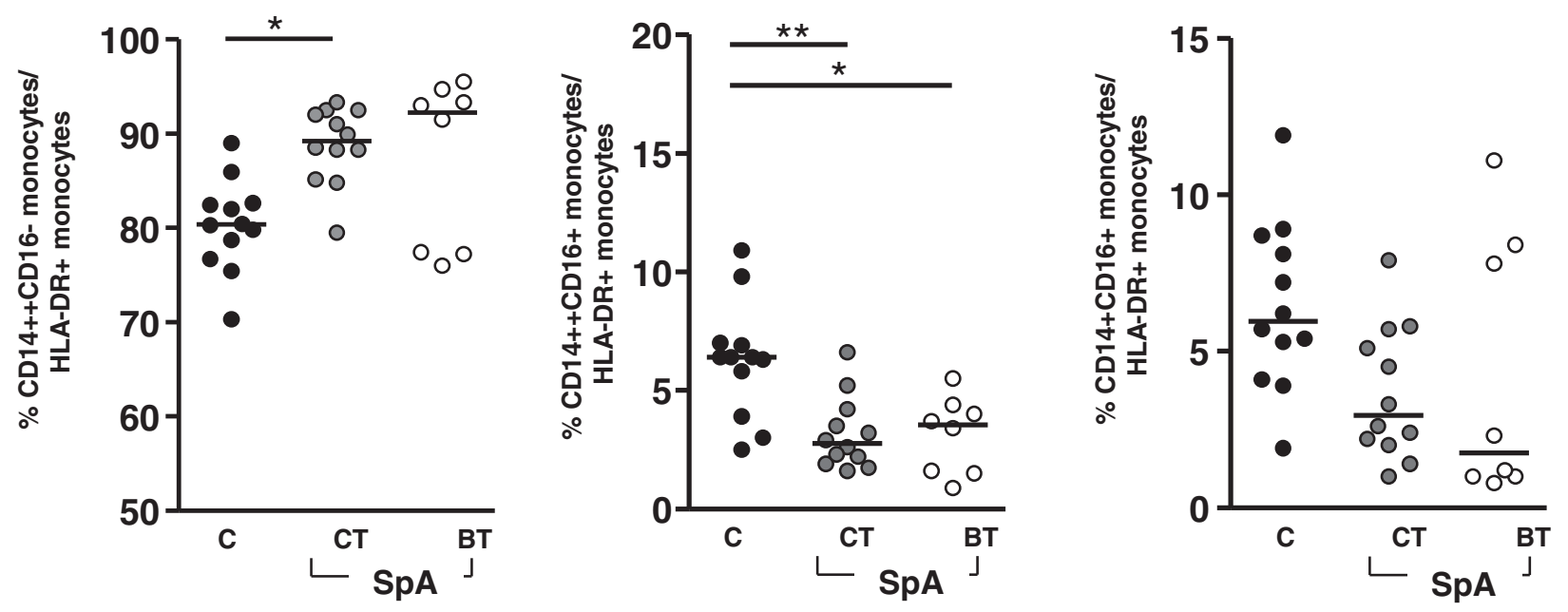

Fig. 1 (See legend on next page.) 
(See figure on previous page.)

Fig. 1 Elevated percentage of classic monocytes in patients with axial spondyloarthritis (axSpA). a The percentage of classic CD14 ${ }^{++} \mathrm{CD} 16^{-}(\mathrm{M} 1)$, intermediate $\mathrm{CD}_{14}{ }^{++} \mathrm{CD} 16^{+}(\mathrm{M} 2)$, and non-classic $\mathrm{CD} 14^{+} \mathrm{CD} 16^{+}(\mathrm{M} 3)$ monocytes was determined by fluorescence-activated cell sorting in whole blood of controls, patients with axSpA, and patients with rheumatoid arthritis (RA). The percentage of classic, intermediate, and non-classic monocytes was determined among monocytes which were gated according to forward (FSC) and side (SSC) scatter (R1) and HLA-DR expression (R2). $\mathbf{b}$ The percentage of classic, intermediate, and non-classic monocytes (individual measurements and median) is shown from measurements in 12 controls (C), 20 patients with axSpA (SpA), and 12 patients with RA (RA). ${ }^{*} P<0.05$, ${ }^{* *} P<0.01$ (Kruskal-Wallis test and Dunn's post test). $\mathbf{c}$ The percentage of the respective populations in axSpA patients separated into patients under conventional treatment (SpA CT) $(n=12)$ and patients receiving biologics (i.e., tumor necrosis factor inhibitors) (SpA BT) $(n=8)$ compared with controls (c). ${ }^{*} P<0.05$, ${ }^{* *} P<0.01$ (Kruskal-Wallis test and Dunn's post test). FITC fluorescein isothiocyanate

elicited higher responses than MDP, FSL-1, or $\mathrm{PAM}_{3} \mathrm{CSK}_{4}$ as indicated by the higher mean fluorescence intensity of the IL-1 $\beta$ staining (Fig. $2 a$ ).

However, LPS stimulation resulted in significantly less IL-6-producing monocytes in axSpA patients compared with controls (median of $63.2 \%$ versus $72.1 \% ; P<0.01$; Fig. 2b). Also, in patients with RA, LPS-induced IL-6 and even TNF $\alpha$ production was reduced in comparison with healthy controls (Fig. 2b). No significant difference between axSpA patients and controls was found in monocytic cytokine production in response to $\mathrm{PAM}_{3} \mathrm{CSK}_{4}$ stimulation (Fig. 2b).

The elevated spontaneous and MDP, and FSL-1, induced proinflammatory cytokine production by monocytes of patients with axSpA suggests in vivo preactivation of monocytes in these patients.

\section{Elevated frequency of monocytes spontaneously producing interleukin-1 receptor antagonist in axial spondyloarthritis and rheumatoid arthritis patients}

To determine whether the preactivation of monocytes in patients with axSpA is counterbalanced by an induction of anti-inflammatory mediators, we analysed the production of IL-1ra and IL-10. IL-1ra was detected by intracellular staining and FACS, whereas IL-10 which is induced at later time points after activation was measured by ELISA in the supernatant of whole blood stimulations performed in the absence of BFA. (Such cultures were performed only in axSpA patients and controls.)

The percentage of monocytes spontaneously producing IL-1ra was significantly elevated in axSpA patients but also RA patients compared with healthy controls (Fig. 3a). In contrast, no difference was found after stimulation with the different stimuli in the percentage of IL1ra-producing monocytes between axSpA or RA patients and controls.

The IL-10 production induced by LPS was significantly lower in axSpA patients compared with controls (median of 1860 versus $3314 \mathrm{pg} / \mathrm{ml} ; P<0.05)$. In contrast, no difference was found in spontaneous or MDP-, FSL-1-, or $\mathrm{PAM}_{3} \mathrm{CSK}_{4}$-induced IL-10 production between axSpA patients and controls (Fig. 3).
Analysis of association of monocytic cytokine production with clinical parameters and treatment in patients with axial spondyloarthritis

To determine the association between cytokine production and the state of disease progression according to the presence of radiographic sacroiliitis or the treatment, we log-transformed the data of patients with axSpA and performed a univariate variance analysis. This analysis (ANOVA) revealed association of spontaneous cytokine production (IL-1 $\beta, P=0.009 ; \quad \mathrm{IL}-6, \quad P=0.03 ; \quad \mathrm{TNF} \alpha$, $P=0.012)$ and FSL-1-induced IL-6 production $(P=0.007)$ with therapy-conventional treatment versus treatment with biologics (i.e., TNF $\alpha$ inhibitors) - but no association with disease state (i.e., presence of radiographic sacroiliitis).

\section{Monocytic pre-activation is confined to axial spondyloarthritis patients under conventional treatment} As the previous analysis suggested an impact of the therapy on the cytokine responses by monocytes, we performed a subanalysis of the data by stratifying the axSpA patients according to therapy into patients receiving conventional treatment and patients receiving TNF $\alpha$ inhibitors. This subanalysis revealed that elevated spontaneous production of IL-1 $\beta$, IL- 6 , and TNF $\alpha$ was confined to axSpA patients under conventional treatment (Fig. 4). In this group, the percentage of monocytes spontaneously producing IL- $1 \beta$ and TNF $\alpha$ was significantly higher than in patients under TNF $\alpha$ inhibitor (i.e., biological treatment $)(P<0.05)$.

Also, the enhanced production of proinflammatory cytokines in response to MDP and FSL-1 was restricted to the group of patients under conventional treatment (Fig. 4). In this group of patients, the percentage of monocytes producing IL-1 $\beta$ and IL- 6 after MDP and FSL-1 stimulation was significantly higher than in controls even after subtraction of the percentage of monocytes spontaneously producing these cytokines (data not shown). In contrast, no difference was found between patients under conventional treatment and patients under TNF $\alpha$ inhibitor treatment in the percentage of monocytes producing IL-12/23p40 (spontaneous or MDPor FSL-induced). 


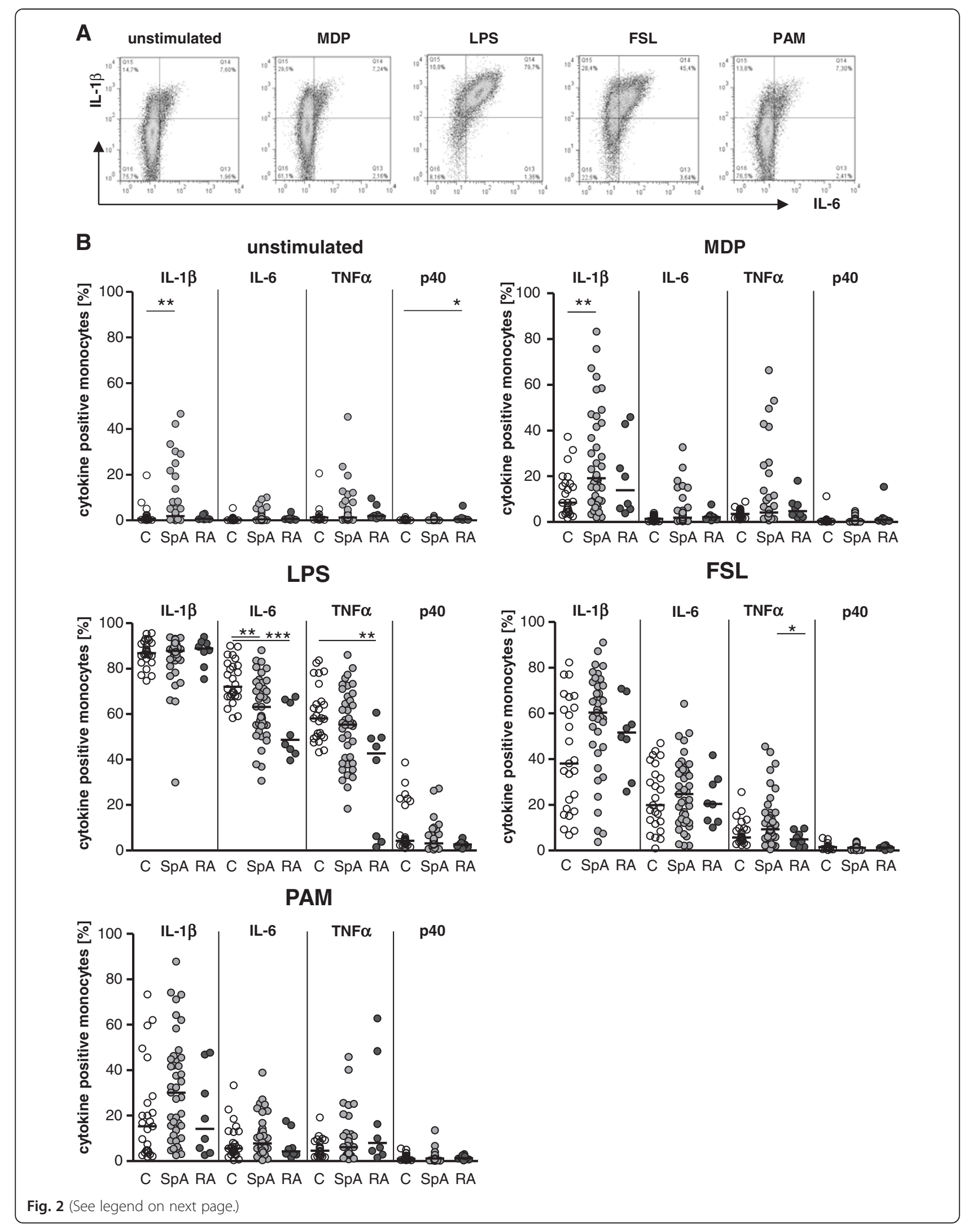


(See figure on previous page.)

Fig. 2 Elevated spontaneous and muramyl dipeptide (MDP)-induced proinflammatory cytokine production by monocytes from patients with axial spondyloarthritis (axSpA). Cytokine production by monocytes after stimulation of whole blood without (unstimulated) or with MDP, lipopolysaccharide (LPS), fibroblast-stimulating lipopeptid-1 (FSL), and PAM $\mathrm{CSK}_{4}$ (PAM) for $5 \mathrm{~h}$ with brefeldin A added for the last 3 h. Cytokine production was analysed after intracellular staining by fluorescence-activated cell sorting. Monocytes were identified according to CD68 expression. a A representative example of cytokine production of $\mathrm{CD}^{+} 8^{+}$monocytes of a patient with axSpA in whole blood without stimulation and stimulation with the respective stimuli is shown. $\mathbf{b}$ The percentage of cytokine-positive cells among CD68 ${ }^{+}$monocytes (individual measurement and median) in response to the different stimuli in controls (C) $(n=26)$, patients with axSpA (SpA) $(n=42)$, and patients with rheumatoid arthritis (RA) $(n=8)$ is given. ${ }^{*} P<0.05$; ${ }^{* *} P<0.01$ (Kruskal-Wallis test and Dunn's post test). IL interleukin, TNFa tumour necrosis factor alpha
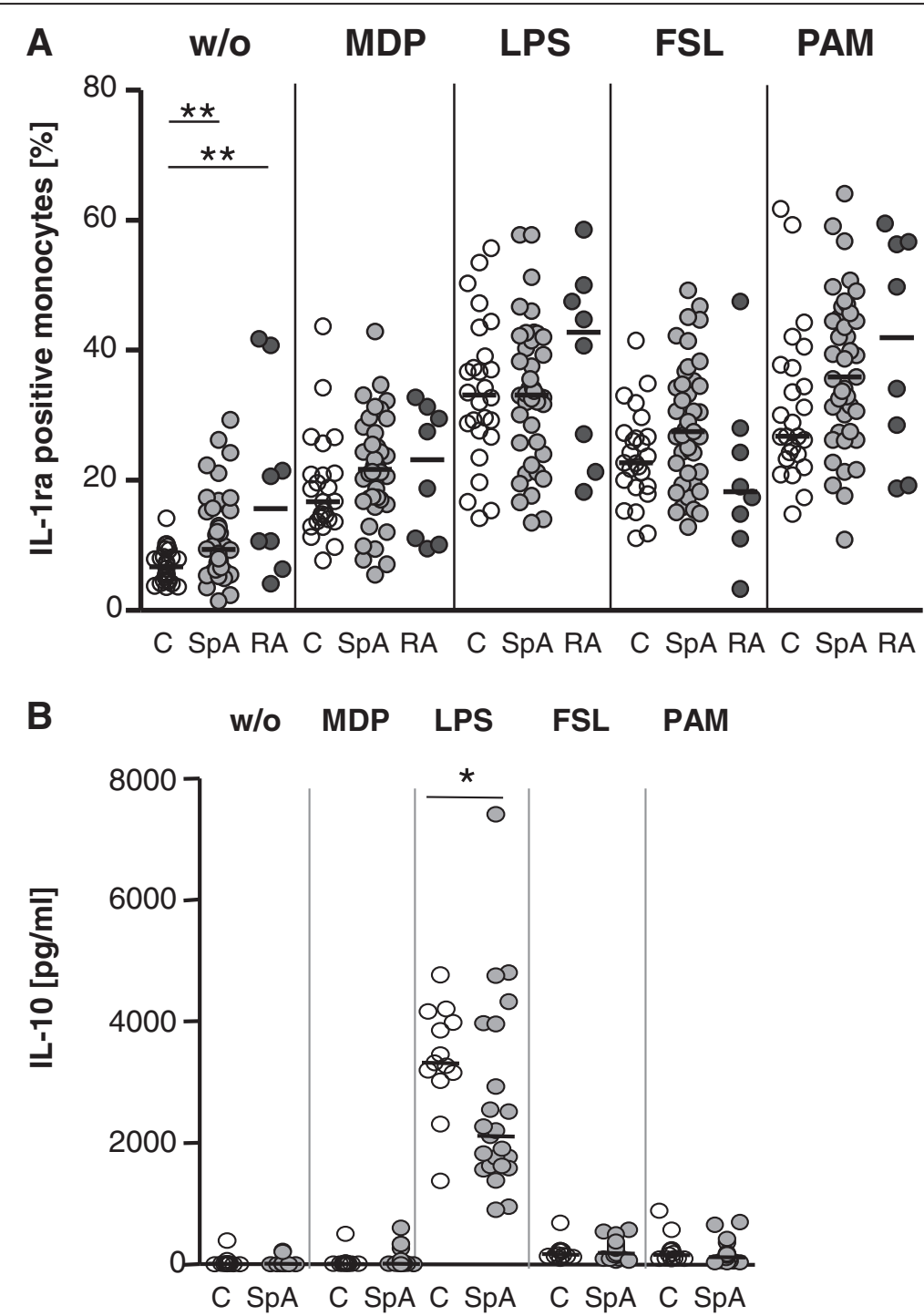

Fig. 3 a Elevated spontaneous production of interleukin-1 receptor antagonist (IL-1ra) by monocytes of patients with axial spondyloarthritis (axSpA) and rheumatoid arthritis (RA). The percentage of IL-1 ra-producing monocytes among CD68 ${ }^{+}$monocytes was determined as in Fig. 2. The percentage of cytokine-positive cells (individual measurement and median) in response to the different stimuli in controls (C) $(n=26)$, patients with axSpA (SpA) $(n=42)$, and patients with RA (RA) $(n=8)$ is given. ${ }^{*} P<0.01$ (Kruskal-Wallis test and Dunn's post test). Whole blood of patients with axSpA (SpA) $(n=26)$ and controls (C) $(n=13)$ was stimulated without or with indicated stimulators for $18 \mathrm{~h}$. $\mathbf{b} \mathrm{LL}-10$ was measured in the supernatant by enzyme-linked immunosorbent assay. ${ }^{*} P<0.05$ (Mann-Whitney U test). FSL fibroblast-stimulating lipopeptid-1, LPS lipopolysaccharide, MDP muramyl dipeptide, PAM PAM $\mathrm{CSK}_{4}$, w/o without 

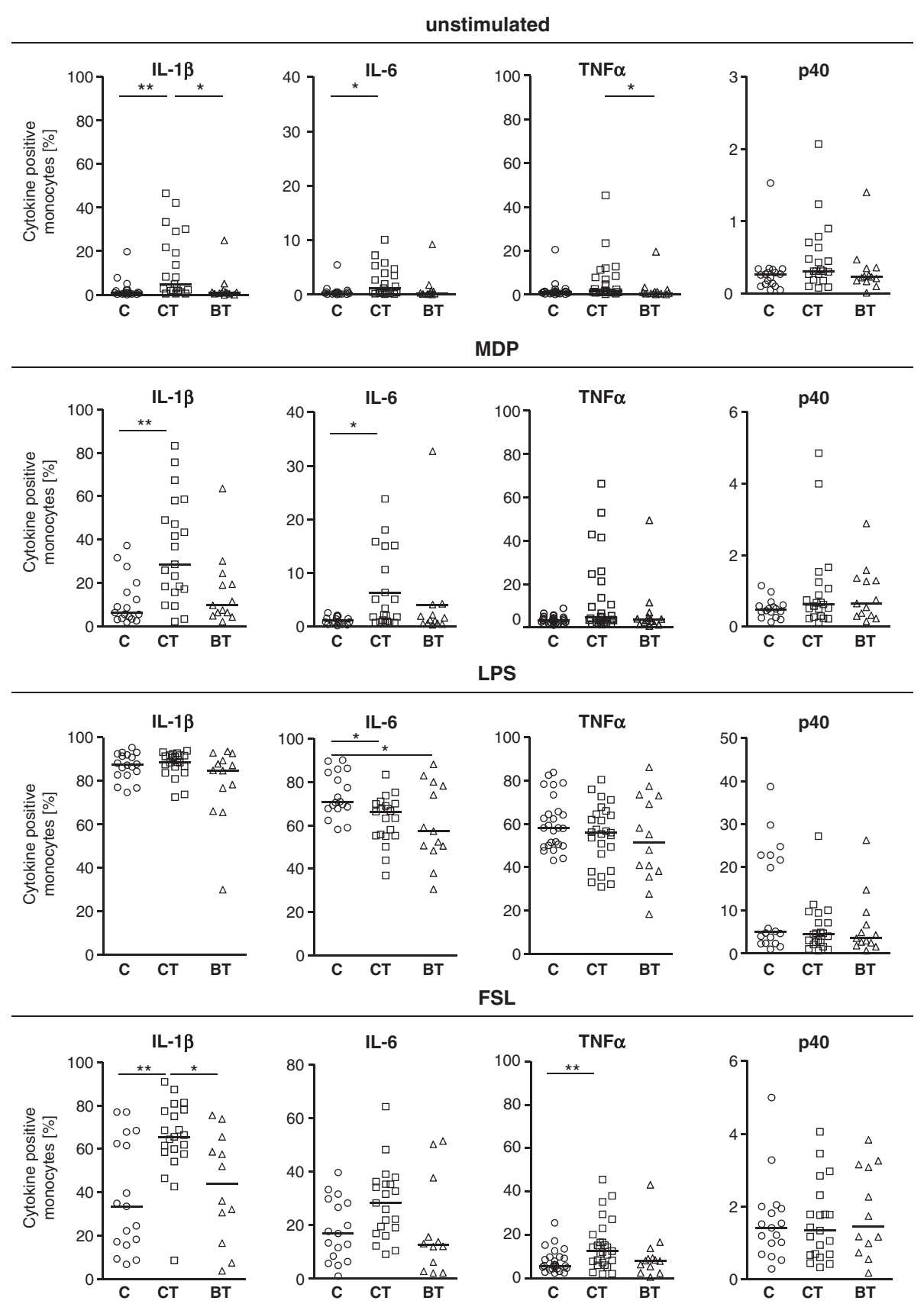

Fig. 4 Disturbed cytokine responses are found predominately in axial spondyloarthritis patients under conventional treatment. Subanalysis of monocytic cytokine production (Fig. 3) in patients stratified according to treatment into a group of patients receiving conventional treatment spondyloarthritis (CT) $(n=20)$ and patients receiving biological treatment (i.e., TNF inhibitor treatment) (BT) $(n=12)$ compared with healthy controls (C) $(n=17) .{ }^{*} P<0.05 ;{ }^{*} P<0.01$ (Kruskal-Wallis test and Dunn's post test). FSL fibroblast-stimulating lipopeptid-1, IL interleukin, LPS lipopolysaccharide, MDP muramyl dipeptide, TNFa tumour necrosis factor alpha

Interestingly, LPS-induced cytokine responses did not differ between both groups of patients; that is, IL-6 (Fig. 4) and IL-10 production (data not shown) was reduced in both groups of patients compared with controls $(P<0.05)$. Moreover, there was no difference in the percentage of IL-1ra-producing monocytes between patients under conventional treatment and patients under TNF $\alpha$ inhibitor treatment (data not shown).

To determine the association between monocytic cytokine production and clinical parameters such as inflammatory parameters, presence of radiographic sacroiliitis, and disease activity in patients with axSpA under conventional 
treatment, we performed an ANOVA and linear regression analysis. In this analysis, monocytic cytokine production (unstimulated and MDP-, LPS-, and FSL-1-stimulated) was not associated with CRP, but a strong association of monocytic cytokine production was observed with BASDAI (Table 1).

\section{Difference in cytokine production by monocytes is not due to differences in Toll-like receptor expression in patients with axial spondyloarthritis}

To determine whether differences in TLR ligand-induced monocytic cytokine production between axSpA patients and controls are due to differences in expression of TLR ligands, we analysed the expression of TLR 1, 2, and 4 on monocytes. No difference was found in surface expression

Table $1 P$ values from univariate analysis of variance between clinical variables and cytokine responses in axial spondyloarthritis patients $(n=20)$ under standard treatment ( $P$ values, log-transformed data)

\begin{tabular}{|c|c|c|c|}
\hline & Sacroiliitis & CRP & BASDAI \\
\hline \multicolumn{4}{|c|}{ Unstimulated } \\
\hline $\mid \mathrm{L}-1$ & 0.980 & 0.539 & 0.011 \\
\hline IL-6 & 0.604 & 0.829 & 0.025 \\
\hline TNF & 0.856 & 0.984 & 0.006 \\
\hline IL-1RA & 0.299 & 0.449 & 0.181 \\
\hline $\mid \mathrm{IL}-10$ & 0.488 & 0.584 & 0.318 \\
\hline \multicolumn{4}{|l|}{ MDP } \\
\hline $\mid \mathrm{L}-1$ & 0.300 & 0.126 & 0.040 \\
\hline IL-6 & 0.693 & 0.698 & 0.034 \\
\hline TNF & 0.439 & 0.963 & 0.007 \\
\hline IL-1RA & 0.098 & 0.433 & 0.010 \\
\hline $\mid \mathrm{L}-10$ & 0.634 & 0.812 & 0.177 \\
\hline \multicolumn{4}{|l|}{ LPS } \\
\hline $\mid \mathrm{L}-1$ & 0.888 & 0.584 & 0.005 \\
\hline IL-6 & 0.546 & 0.625 & 0.029 \\
\hline TNF & 0.692 & 0.516 & 0.001 \\
\hline IL-1RA & 0.543 & 0.744 & 0.048 \\
\hline$\| \mathrm{L}-10$ & 0.691 & 0.742 & 0.010 \\
\hline \multicolumn{4}{|l|}{ FSL } \\
\hline $\mid \mathrm{L}-1$ & 0.492 & 0.470 & 0.642 \\
\hline IL-6 & 0.389 & 0.483 & 0.669 \\
\hline TNF & 0.861 & 0.918 & 0.669 \\
\hline IL-1RA & 0.227 & 0.390 & 0.040 \\
\hline $\mid \mathrm{L}-10$ & 0.714 & 0.199 & 0.037 \\
\hline
\end{tabular}

CRP C-reactive protein, BASDAl Bath ankylosing spondylitis disease activity index, IL interleukin, TNF tumor necrosis factor, MDP muramyl dipeptide, LPS lipopolysaccharide, FSL fibroblast-stimulating lipopeptid of either of the receptors on monocytes in axSpA patients and RA patients compared with controls (Fig. 5).

\section{Discussion}

In this study, we observed an elevated frequency of classic (i.e., $\mathrm{CD} 14^{++} \mathrm{CD} 16^{-}$) monocytes and a reduction in non-classic (i.e., $\mathrm{CD} 14^{+} \mathrm{CD} 16^{+}$) monocytes in peripheral blood of patients with axSpA and this is in accordance with a study by Surdacki et al. [11]. TNF $\alpha$ inhibitor treatment did not reverse these phenotypic differences. In patients with RA, we also observed a trend toward an increase of classic monocytes at the expense of nonclassic monocytes. This is in line with data from Klimek et al. [12] but in contrast to other studies which reported an expansion of CD14/CD16 double-positive (i.e., nonclassic) monocytes in RA [13, 14]. These discrepancies are most likely related to differences in therapy; in particular, glucocorticoid therapy has been described to deplete $\mathrm{CD} 14^{+} \mathrm{CD} 16^{+}$monocytes [15]. In patients with Crohn's disease, an expansion of non-classic monocytes was described [16]. It was suggested that the imbalance between monocyte subsets in patients with Crohn's disease is due to recruitment of the classic monocytes, which are more migratory than the non-classic monocytes, to sites of inflammation [16]. Therefore, the difference between axSpA and Crohn's disease may be caused by differences in the extent of local inflammation which controls recruitment of monocytes. On the other hand, HLA-B27 could also affect the differentiation of monocyte subsets and promote the differentiation of classic monocytes.

Thiesen et al. analysed cytokine production of sorted human monocyte subsets and found that classic monocytes, though considered more phagocytic and less inflammatory, produced higher levels of proinflammatory cytokines than the non-classic monocytes [16]. Interestingly, we observed enhanced spontaneous as well as MDP- and FSL-1-induced production of proinflammatory cytokines in patients with axSpA and, according to the data by Thiesen et al., this could be related to the expansion of classic monocytes.

The enhanced cytokine production by monocytes of patients with axSpA in particular to suboptimal stimuli such as MDP and FSL-1, which require co-activating signals such as ineffective doses of LPS [17] or low levels of cytokines, suggests that monocytes of patients with axSpA are pre-activated in vivo. This is also suggested by mRNA expression profiles showing enhanced IL-1 receptor expression in blood cells of patients with axSpA [18] and by results of protein profiling of monocytes of axSpA patients that showed enhanced expression of proteins involved in TLR signaling, leukocyte extravasation, and other inflammatory pathways [8]. Genome association studies [19] and experimental [20] and clinical [21] data suggest an involvement of the IL-23-Th17 pathway 
TLR-1

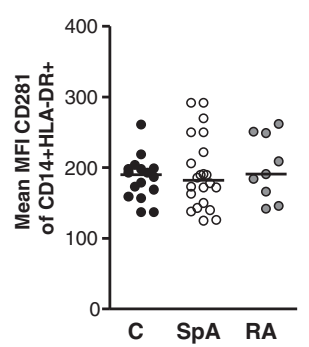

TLR-2

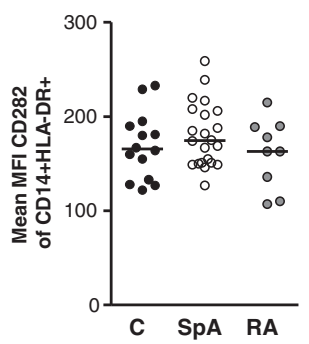

TLR-4

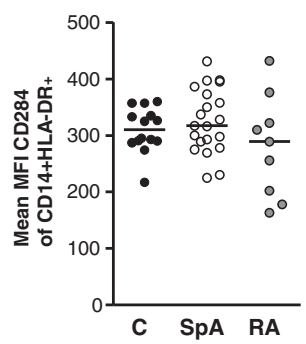

Fig. 5 No difference in Toll-like receptor (TLR) 1, 2, and 4 expression on CD14 ${ }^{+}$monocytes between axial spondyloarthritis patients and controls. The expression of TLR-1, TLR-2, and TLR-4 on monocytes was determined in healthy controls (C) $(n=16)$, patients with axial spondyloarthritis $(\mathrm{SpA})(n=22)$, and patients with rheumatoid arthritis (RA) $(n=9)$ by surface staining and fluorescence-activated cell sorting and is given as the mean fluorescence intensity (MFI). Monocytes were identified according to CD14 expression

in axSpA. However, the production of IL-12/23p40 production was rather low in peripheral blood monocytes in our study and with no difference between axSpA patients and controls. Even after strong stimulation with LPS, only about $5 \%$ of the monocytes produced IL-12/ 23 p40, suggesting that monocytes are not a major source of this cytokine. In fact, our own analysis of the bone marrow of facet joints of patients with AS revealed myeloperoxidase-positive cells and, to a lesser extent, macrophages and dendritic cells as sources of IL-23 [22].

In contrast to the enhanced cytokine responses to MDP and FSL-1, cytokine production in response to LPS was, at least partially, reduced in patients with axSpA. Reduced LPS responses are observed in endotoxin tolerance which is induced by pre-exposure of monocytes to low doses of LPS. However, the suppression of proinflammatory cytokine responses by monocytes of patients with axSpA is rather minor and accompanied by a strong suppression of anti-inflammatory IL-10 production, whereas the state of endotoxin tolerance and monocyte paralysis, as for instance observed in patients with sepsis, is characterized by a strongly suppressed proinflammatory response and partly preserved IL-10 production [23]. Thus, the decent reduction of cytokine responses to LPS which is the strongest of the tested stimuli may indicate exhaustion of monocytes after pre-activation rather than tolerization.

The enhanced spontaneous production of cytokines was restricted to axSpA patients under conventional treatment and is not found in axSpA patients under TNF $\alpha$ inhibitor treatment or in RA patients. However, LPS-induced IL-6 and IL-10 production was also reduced in axSpA patients who received TNF $\alpha$ inhibitor. This could be attributable to the presence of TNFa inhibitors in the whole blood stimulation which may neutralize some autocrine effects of TNF $\alpha$ produced during early activation. In particular, IL-10 depends on autocrine production of TNFa [7]. Also, in patients with RA, LPS-induced production of IL- 6 and TNF $\alpha$ was reduced and this may also be related to glucocorticoid therapy [24].
Differences in cytokine responses to TLR ligands may be caused by differential expression of TLRs. In fact, enhanced expression of TLR 4 on blood monocytes has been described in patients with SpA [25]. However, in our study, no differences were found in the expression of TLR 1,2, and 4 in axSpA patients compared with controls and this might be due to technical differences as we analysed expression in whole blood as compared with isolated peripheral blood mononuclear cells and used a different gating strategy.

Interestingly, stimulation of bone marrow cells and splenocytes from HLA-B27/hu $\beta 2 \mathrm{~m}$ transgenic rats with TNF $\alpha$ [26] or zymosan or Mycobacterium tuberculosis also resulted in elevated production of IL- $1 \alpha$ and IL- $1 \beta$ compared with control rats, indicating that the enhanced responsiveness of axSpA patients to bacterial triggers may be related to HLA-B27. On the other hand, the expansion of classic monocytes and in vivo pre-activation of monocytes in patients with axSpA could also be the result of in vivo activation by cytokines released by local inflammation or by bacterial components such as LPS which may translocate during intestinal inflammation as mucosal inflammation is a common feature in axSpA. Thus, about $5 \%$ of patients with AS also suffer from overt inflammatory bowel disease [27] and about $50 \%$ show microscopic gut lesions in the absence of gastrointestinal discomfort [28].

Delineation of potential triggers of the spontaneous cytokine production is difficult since cellular responses of monocytes to LPS and cytokines such as TNF $\alpha$ are strongly overlapping [29]. Only some genes such as apoptosis-related genes are selectively induced in monocytes by LPS in vitro [29] and analysis of their expression might provide clues on the activating signal of monocytes in patients with axSpA.

TNF $\alpha$ inhibitor treatment of axSpA normalized the functional state of monocytes as elevated cytokine responses were confined to axSpA patients under conventional treatment. The fact that MDP- and FSL-1-induced 
cytokine production was unaffected under TNF $\alpha$ inhibitor treatment suggests that the effect of TNF $\alpha$ inhibitor treatment is an in vivo effect rather than an in vitro effect. Thus, the TNF $\alpha$ inhibitor treatment may interfere with the triggers of monocytic cytokine production such as local cytokine production or bacterial antigen translocation.

Interestingly, we found that spontaneous MDP- and LPS-induced production of cytokines correlated with disease activity. As CRP correlated neither with BASDAI nor with the monocytic cytokine production, monocyte activation might be a more sensitive indicator of inflammation than CRP. However, the monocytic pre-activation, particularly the spontaneous production of IL- $1 \beta$ as well as IL-6, does not seem to be directly pathogenic as blockade of IL-1 [30] as well as IL-6 [31] is of limited or no efficacy in reducing disease activity in AS. The enhanced IL- $1 \beta$ and IL- 6 production by monocytes rather seems to be an indicator of systemic innate immune activation while other immune functions of monocytes or other cell types may exert the pathogenic effects.

\section{Conclusions}

This study reveals in vivo pre-activation of monocytes in axSpA patients indicated by enhanced spontaneous as well as MDP- and FSL-induced cytokine production by monocytes which correlates with BASDAI. This in vivo pre-activation is confined to patients under conventional therapy and reverted under TNF $\alpha$ inhibitor treatment. Further work should delineate the triggers of this systemic activation of innate cells.

\footnotetext{
Abbreviations

ANOVA: Analysis of variance; AS: Ankylosing spondylitis; axSpA: Axial spondyloarthritis; BASDAl: Bath ankylosing spondylitis disease activity index; BFA: Brefeldin A; CRP: C-reactive protein; DMARD: Disease-modifying anti-rheumatic drug; ELISA: Enzyme-linked immunosorbent assay; FACS: Fluorescence-activated cell sorting; FITC: Fluorescein isothiocyanate; FSL-1: Fibroblast-stimulating lipopeptid-1; IL: Interleukin; LPS: Lipopolysacchride; MDP: Muramyl dipeptide; NOD: Nucleotide oligomerization domain; NSAID: Non-steroidal anti-inflammatory drug; RA: Rheumatoid arthritis; TLR: Toll-like-receptor; TNFa: Tumour necrosis factor alpha.
}

\section{Competing interests}

The authors declare that they have no competing interests.

\section{Authors' contributions}

US and JS helped to design the study, draft the manuscript, and analyse the data. KC helped to perform the assays, collect the data, analyse the data, and draft the manuscript. PW helped to perform the assays, collect the data, and draft the manuscript. All authors read and approved the final manuscript.

\footnotetext{
Acknowledgements

This work was supported by the German Federal Ministry of Education and Research (Bundesministerium für Bildung und Forschung, or BMBF) through the ArthroMark project. We thank all patients for participation in the study and doctors and study nurses in the rheumatology department at the Charité, Campus Benjamin Franklin, for acquisition of samples.
}

Received: 7 January 2015 Accepted: 19 June 2015

Published online: 16 July 2015

\section{References}

1. Braun J, Sieper J. Ankylosing spondylitis. Lancet. 2007;369:1379-90. doi:10.1016/S0140-6736(07)60635-7.

2. May E, Dorris ML, Satumtira N, Iqbal I, Rehman MI, Lightfoot E, et al. CD8 alpha beta $T$ cells are not essential to the pathogenesis of arthritis or colitis in HLA-B27 transgenic rats. J Immunol. 2003;170:1099-105.

3. Colbert RA, DeLay ML, Klenk El, Layh-Schmitt G. From HLA-B27 to spondyloarthritis: a journey through the ER. Immunol Rev. 2010;233:181-202. doi:10.1111/j.0105-2896.2009.00865.x.

4. Freudenberg MA, Galanos C. Bacterial lipopolysaccharides: structure, metabolism and mechanisms of action. Int Rev Immunol. 1990;6:207-21.

5. Beutler B, Kruys V. Lipopolysaccharide signal transduction, regulation of tumor necrosis factor biosynthesis, and signaling by tumor necrosis factor itself. J Cardiovasc Pharmacol. 1995;25:S1-8.

6. Garlanda C, Dinarello CA, Mantovani A. The interleukin-1 family: back to the future. Immunity. 2013;39:1003-18. doi:10.1016/j.immuni.2013.11.010.

7. Platzer C, Meisel C, Vogt K, Platzer M, Volk HD. Up-regulation of monocytic IL-10 by tumor necrosis factor-alpha and CAMP elevating drugs. Int Immunol. 1995;7:517-23.

8. Wright C, Edelmann M, di Gleria K, Kollnberger S, Kramer H, McGowan S, et al. Ankylosing spondylitis monocytes show upregulation of proteins involved in inflammation and the ubiquitin proteasome pathway. Ann Rheum Dis. 2009;68:1626-32. doi:10.1136/ard.2008.097204.

9. Gu J, Marker-Hermann E, Baeten D, Tsai WC, Gladman D, Xiong M, et al. A 588-gene microarray analysis of the peripheral blood mononuclear cells of spondyloarthropathy patients. Rheumatology (Oxford). 2002;41:759-66.

10. Garrett S, Jenkinson T, Kennedy LG, Whitelock H, Gaisford P, Calin A. A new approach to defining disease status in ankylosing spondylitis: the Bath Ankylosing Spondylitis Disease Activity Index. J Rheumatol. 1994;21:2286-91.

11. Surdacki A, Sulicka J, Korkosz M, Mikolajczyk T, Telesinska-Jasiowka D, Klimek $E$, et al. Blood monocyte heterogeneity and markers of endothelial activation in ankylosing spondylitis. J Rheumatol. 2014;41:481-9. doi:10.3899/jrheum.130803

12. Klimek E, Mikolajczyk T, Sulicka J, Kwasny-Krochin B, Korkosz M, Osmenda G, et al. Blood monocyte subsets and selected cardiovascular risk markers in rheumatoid arthritis of short duration in relation to disease activity. Biomed Res Int. 2014;2014:736853. doi:10.1155/2014/736853.

13. Kawanaka N, Yamamura M, Aita T, Morita Y, Okamoto A, Kawashima M, et al. CD14+, CD16+ blood monocytes and joint inflammation in rheumatoid arthritis. Arthritis Rheum. 2002;46:2578-86. doi:10.1002/art.10545.

14. Rossol M, Kraus S, Pierer M, Baerwald C, Wagner U. The CD14(bright) CD16+ monocyte subset is expanded in rheumatoid arthritis and promotes expansion of the Th17 cell population. Arthritis Rheum. 2012;64:671-7. doi:10.1002/art.33418.

15. Dayyani F, Belge KU, Frankenberger M, Mack M, Berki T, Ziegler-Heitbrock L. Mechanism of glucocorticoid-induced depletion of human CD14 + CD16+ monocytes. J Leukoc Biol. 2003;74:33-9.

16. Thiesen S, Janciauskiene S, Uronen-Hansson H, Agace W, Hogerkorp CM, Spee $P$, et al. CD14(hi)HLA-DR(dim) macrophages, with a resemblance to classic blood monocytes, dominate inflamed mucosa in Crohn's disease. J Leukoc Biol. 2014;95:531-41. doi:10.1189/jlb.0113021.

17. Fritz JH, Girardin SE, Fitting C, Werts C, Mengin-Lecreulx D, Caroff M, et al. Synergistic stimulation of human monocytes and dendritic cells by Toll-like receptor 4 and NOD1- and NOD2-activating agonists. Eur J Immunol. 2005;35:2459-70. doi:10.1002/eji.200526286.

18. Sharma SM, Choi D, Planck SR, Harrington CA, Austin CR, Lewis JA, et al. Insights in to the pathogenesis of axial spondyloarthropathy based on gene expression profiles. Arthritis Res Ther. 2009;11:R168. doi:10.1186/ar2855.

19. Cortes A, Hadler J, Pointon JP, Robinson PC, Karaderi T, Leo P, et al. Identification of multiple risk variants for ankylosing spondylitis through high-density genotyping of immune-related loci. Nat Genet. 2013;45:730-8. doi:10.1038/ng.2667.

20. Sherlock JP, Joyce-Shaikh B, Turner SP, Chao CC, Sathe M, Grein J, et al. IL-23 induces spondyloarthropathy by acting on ROR-gammat + CD3 + CD4-CD8entheseal resident T cells. Nat Med. 2012;18:1069-76. doi:10.1038/nm.2817.

21. Poddubnyy D, Hermann KG, Callhoff J, Listing J, Sieper J. Ustekinumab for the treatment of patients with active ankylosing spondylitis: results of a 28-week, prospective, open-label, proof-of-concept study (TOPAS). Ann Rheum Dis. 2014;73:817-23. doi:10.1136/annrheumdis-2013-204248.

22. Appel H, Maier R, Bleil J, Hempfing A, Loddenkemper C, Schlichting U, et al. In situ analysis of interleukin-23- and interleukin-12-positive cells in the spine of patients with ankylosing spondylitis. Arthritis Rheum. 2013;65:1522-9. doi:10.1002/art.37937 
23. Randow F, Syrbe U, Meisel C, Krausch D, Zuckermann H, Platzer C, et al. Mechanism of endotoxin desensitization: involvement of interleukin 10 and transforming growth factor beta. J Exp Med. 1995;181:1887-92.

24. Boumpas DT, Paliogianni F, Anastassiou ED, Balow JE. Glucocorticosteroid action on the immune system: molecular and cellular aspects. Clin Exp Rheumatol. 1991;9:413-23.

25. De Rycke L, Vandooren B, Kruithof E, De Keyser F, Veys EM, Baeten D. Tumor necrosis factor alpha blockade treatment down-modulates the increased systemic and local expression of Toll-like receptor 2 and Toll-like receptor 4 in spondylarthropathy. Arthritis Rheum. 2005;52:2146-58. doi:10.1002/art.21155.

26. Layh-Schmitt G, Yang EY, Kwon G, Colbert RA. HLA-B27 alters the response to tumor necrosis factor alpha and promotes osteoclastogenesis in bone marrow monocytes from HLA-B27-transgenic rats. Arthritis Rheum. 2013;65:2123-31. doi:10.1002/art.38001.

27. Stolwijk C, van Tubergen A, Castillo-Ortiz JD, Boonen A. Prevalence of extra-articular manifestations in patients with ankylosing spondylitis: a systematic review and meta-analysis. Ann Rheum Dis. 2015;74:65-73. doi:10.1136/annrheumdis-2013-203582.

28. Van Praet L, Van den Bosch FE, Jacques P, Carron P, Jans L, Colman R, et al, Microscopic gut inflammation in axial spondyloarthritis: a multiparametric predictive model. Ann Rheum Dis. 2013;72:414-7. doi:10.1136/annrheumdis2012-202135.

29. Smiljanovic B, Grun JR, Steinbrich-Zollner M, Stuhlmuller B, Haupl T, Burmester GR, et al. Defining TNF-alpha- and LPS-induced gene signatures in monocytes to unravel the complexity of peripheral blood transcriptomes in health and disease. J Mol Med (Berl). 2010;88:1065-79. doi:10.1007/ s00109-010-0648-8.

30. Haibel H, Rudwaleit M, Listing J, Sieper J. Open label trial of anakinra in active ankylosing spondylitis over 24 weeks. Ann Rheum Dis. 2005;64:296-8. doi:10.1136/ard.2004.023176.

31. Sieper J, Braun J, Kay J, Badalamenti S, Radin AR, Jiao L, et al. Sarilumab for the treatment of ankylosing spondylitis: results of a Phase II, randomised, double-blind, placebo-controlled study (ALIGN). Ann Rheum Dis. 2015;74:1051-7. doi:10.1136/annrheumdis-2013-204963.

\section{Submit your next manuscript to BioMed Central and take full advantage of:}

- Convenient online submission

- Thorough peer review

- No space constraints or color figure charges

- Immediate publication on acceptance

- Inclusion in PubMed, CAS, Scopus and Google Scholar

- Research which is freely available for redistribution 